\title{
Student Exercises on Fossil Fuels, Global Warming, and Gaia
}

\author{
Bert W. Rust \\ National Institute of Standards and Technology, 100 Bureau Drive, Stop 8910, \\ Gaithersburg, MD 20899-8910, USA \\ bert.rust@nist.gov
}

\begin{abstract}
In a recent series of tutorial papers, Rust 78 8.910 modelled measured time series for global temperatures and fossil fuel $\mathrm{CO}_{2}$ emissions with related combinations of polynomials, exponentials, and sinusoids, using least squares fits that can be done by students well grounded in practical statistics. The analysis suggested that temperatures cycle around a monotonically increasing, accelerating baseline with a period of $\approx 65$ years and that the exponential growth rate of $\mathrm{CO}_{2}$ emissions varies inversely with this cycle. The Gaia hypothesis [5] suggests that the biosphere adjusts atmospheric greenhouse gases to maintain an optimal temperature for life. The previous analysis is here extended with a series of fitting exercises designed to demonstrate that the above described inverse relation represents a Gaian feedback.
\end{abstract}

\section{Introduction}

Figure 1 gives a plot of annual global total fossil fuel $\mathrm{CO}_{2}$ emissions, measured in millions of metric tons of carbon [MtC], for the years 1856-1999. These data, which were compiled by Marland, et. al. [6], can be found at http://cdiac.ornl.gov /trends/emis/em_cont.htm. The dashed curve is a nonlinear fit [9] of the model

$$
P\left(t-t_{0}\right)=P_{0} e^{\alpha\left(t-t_{0}\right)},
$$

where $P(t)$ is the total emission in year $t, t_{0}=1856.0$, and $P_{0}$ and $\alpha$ are free parameters. The observations undulate systematically around the fit, and a Fourier analysis of the residuals indicated a cycle with period $\approx 65$ years. The amplitude of the cycle appeared to increase in time with the same exponential rate as the fitted curve. This suggested a model of the form

$$
P\left(t-t_{0}\right)=\left\{P_{0}+P_{1} \sin \left[\frac{2 \pi}{\tau}\left(t-t_{0}+\theta\right)\right]\right\} e^{\alpha\left(t-t_{0}\right)},
$$

with free parameters $P_{0}, P_{1}, \alpha, \tau, \theta$. Fitting this model [10] gave estimates

$$
\begin{gathered}
\hat{P}_{0}=133.8 \pm 5.0[M t C], \quad \hat{P}_{1}=25.4 \pm 1.6[M t C], \\
\hat{\tau}=64.9 \pm 1.5[\mathrm{yr}], \quad \hat{\theta}=26.6 \pm 2.7[\mathrm{yr}], \quad \hat{\alpha}=0.02814 \pm .00034\left[\mathrm{yr}^{-1}\right] .
\end{gathered}
$$

The fit, plotted as a solid curve, explains $99.73 \%$ of the variance in the data. 


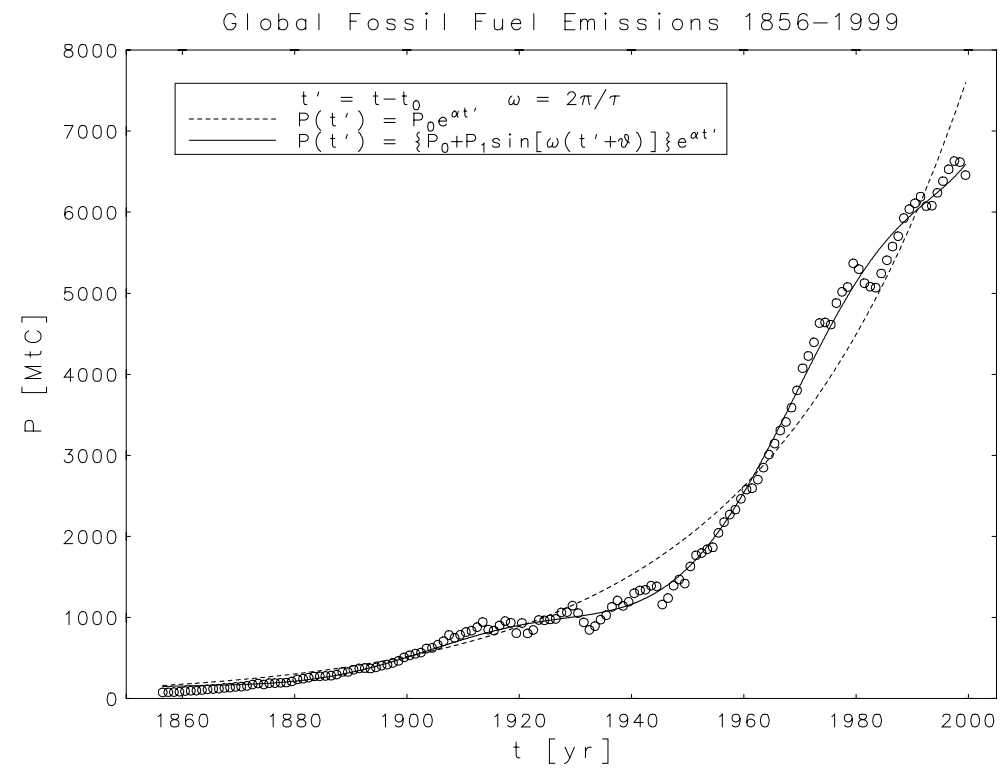

Fig. 1. Annual global total fossil fuel $\mathrm{CO}_{2}$ emissions are plotted as discrete circles. The dashed curve is the fit of the model (1), and the solid curve is the fit of (2).

Figure 2 is a plot of annual global average tropospheric temperature anomalies (in ${ }^{\circ} \mathrm{C}$ ) for the years $1856-2001$. The anomalies were obtained from the measured temperatures by subtracting the average temperature for 1961-1990. These data, which were calculated by P. D. Jones, et. al. [2, can be obtained online at http://www.cru.uea.ac.uk/cru/cru.htm. Low order polynomials fits [7] 8] suggested an accelerating warming with a monotonically increasing baseline. If $T(t)$ is the anomaly in year $t$, then warming with constant acceleration can be modelled by

$$
T\left(t-t_{0}\right)=T_{0}+C_{2}\left(t-t_{0}\right)^{2},
$$

with free parameters $T_{0}$ and $C_{2}$. Including a linear term did not significantly reduce the sum of squared residuals [8]. A slightly better fit was obtained [9] for

$$
T\left(t-t_{0}\right)=T_{0}+C_{2} \exp \left[0.01407\left(t-t_{0}\right)\right],
$$

where the rate $0.01407\left[\mathrm{yr}^{-1}\right]$ is one half of the $\hat{\alpha}$ in (3). The residuals for both fits oscillated with period $\tau \approx 63$ years [8911 which is close to the $\hat{\tau}$ in (3). Also, the oscillation was a half cycle out of phase with the emissions cycle, with maximum emissions corresponding to minimum temperatures, and vice versa. This temperature cycle was previously noted by Schlesinger and Ramankutty [14, and the inverse correlation between variations in temperature and emissions was previously noted by Rust and Kirk [13. Their work was extended by Rust and Crosby 12] who argued that the correlation arises from a Gaian feedback by which increasing temperatures reduce the growth in fossil fuel production. 


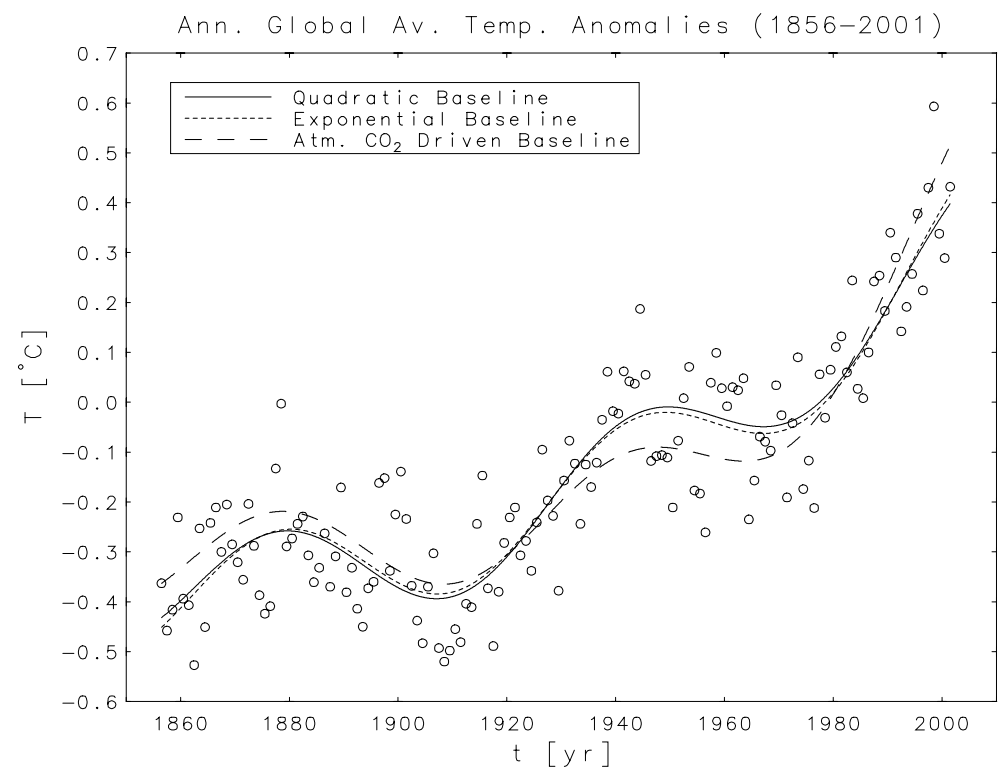

Fig. 2. The circles are observed annual global average temperature anomalies. The solid curve is the fit of the quadratic/sinusoidal model (6), the finely dashed curve is the fit of the exponential/sinusoidal model (7), and the coarsely dashed curve is the fit of the atmospheric- $\mathrm{CO}_{2}$-driven/sinusoidal model (10).

Adding the temperature cycle to the models (44) and (5) gives

$$
T\left(t-t_{0}\right)=T_{0}+C_{2}\left(t-t_{0}\right)^{2}+A_{1} \sin \left[\frac{2 \pi}{64.9}\left(t-t_{0}-5.85\right)\right],
$$

and

$$
T\left(t-t_{0}\right)=T_{0}+C_{2} \exp \left[0.01407\left(t-t_{0}\right)\right]+A_{1} \sin \left[\frac{2 \pi}{64.9}\left(t-t_{0}-5.85\right)\right],
$$

with free parameters $T_{0}, C_{2}$, and $A_{1}$. The period of the sinusoid is exactly the $\hat{\tau}$ in (3), and the phase constant has been set to $\hat{\theta}-(\hat{\tau} / 2)=-5.85$ to make the oscillation exactly one half cycle ahead (or behind) the one for the emissions. Fitting these two models [10] gave the parameter estimates in columns 2 and 3 of Table 1 and the solid and finely dashed curves in Fig. 2.

\section{Exercises 1 and 2: Precedence and Causality}

A 64.9 year cycle in both the temperatures and the emissions cannot be a coincidence. $\mathrm{CO}_{2}$ is a greenhouse gas, so its accumulation in the atmosphere is expected to cause some warming. But is the cycle an emissions effect vectored into the temperatures, or is it a Gaian feedback by which increasing temperatures 
Table 1. Estimates and coefficients of determination for models (6), (7), and (10).

\begin{tabular}{|c|rcr|}
\hline \hline Param. & Quad. Baseline(6) Expon. Baseline (7) $\mathrm{CO}_{2}$-driven Baseline (10) \\
\hline \hline$\hat{T}_{0}$ & $-0.380 \pm .013$ & $-0.510 \pm .017$ & $-3.25 \pm .14$ \\
$\hat{C}_{2}$ & $(3.27 \pm .14) \times 10^{-5}$ & $0.1094 \pm .0045$ & $(4.66 \pm .21) \times 10^{-6}$ \\
$\hat{A}_{1}$ & $0.105 \pm .013$ & $0.104 \pm .013$ & $0.090 \pm .013$ \\
\hline$R^{2}$ & 0.8071 & 0.8081 & 0.7802 \\
\hline \hline
\end{tabular}

supress fossil fuel consumption? One indication of causality would be a definite time delay between the cycles in the two records. If the phase constants -5.85 in (6) and (7) are replaced by free parameters $\phi_{q}$ and $\phi_{e}$, then two 4-parameter linear least squares fits give estimates $\hat{\phi}_{q}=-4.75 \pm 1.17$ and $\hat{\phi}_{e}=-4.80 \pm 1.18$. These estimates indicate a nominal lag of approximately one year between a temperature extremum and the opposite extremum in the emissions. But -5.85 falls inside the $\pm 1 \sigma$ uncertainty intervals for both estimates, so that lag is not statistically significant. Also, for both fits, an F-test indicates that the additional free parameter does not produce a statistically significant reduction in the sum of squared residuals. Thus the data cannot reliably determine precedence for the two cycles.

\section{Exercise 3: Atmospheric Accumulation of $\mathrm{CO}_{2}$}

High quality measurements of global atmospheric $\mathrm{CO}_{2}$ concentrations, made by C. D. Keeling, et. al. [4], can be found at http://cdiac.ornl.gov/ftp/maunaloaco2/maunaloa.co2. Yearly averages for 1959-2001 are plotted in Fig. 3 It is generally accepted that $\approx 50 \%$ of the fossil fuel $\mathrm{CO}_{2}$ emissions remains in the atmosphere, so a model for atmospheric accumulation $c(t)$ can be written

$$
c\left(t-t_{0}\right)=c_{0}+\gamma \int_{0}^{t-t_{0}} P\left(t^{\prime}\right) d t^{\prime}
$$

where $c_{0}$ is the concentration at epoch $1856.0, P\left(t^{\prime}\right)$ is the emissions total in year $t^{\prime}$, and $\gamma$ is the fraction remaining in the atmosphere. Substituting (2) gives

$$
c\left(t-t_{0}\right)=c_{0}+\gamma \int_{0}^{t-t_{0}}\left\{\hat{P}_{0}+\hat{P}_{1} \sin \left[\frac{2 \pi}{\hat{\tau}}\left(t^{\prime}+\hat{\theta}\right)\right]\right\} e^{\hat{\alpha} t^{\prime}} d t^{\prime},
$$

where $\hat{P}_{0}, \hat{P}_{1}, \hat{\tau}, \hat{\theta}$, and $\hat{\alpha}$ are the estimates given in (3). It is an easy linear least squares exercise to fit this model, with free parameters $c_{0}$ and $\gamma$, to Keeling's 
data. The integral could be evaluated in closed form, but it is easier to compute it with a numerical integrator. The only complication is that the units of the atmospheric measurements are [ppmv], but the units of $\hat{P}_{0}$ and $\hat{P}_{1}$ are [MtC]. The conversion factor [15] is $1[\mathrm{ppmv}]=2.130 \times 10^{3}[\mathrm{MtC}]$. The strategy used here was to convert the measurements to $[\mathrm{MtC}]$ to obtain the estimates $\hat{c}_{0}=$ $(6.2899 \pm .0058) \times 10^{5}[\mathrm{MtC}]$ and $\hat{\gamma}=0.572 \pm .032$. The fit, which explained $99.87 \%$ of the variance, was converted back to [ppmv] units to be plotted as the smooth curve in Fig. 3. Clearly the 64.9 year cycle was totally smoothed out by the cumulative quadrature, so its appearance in the temperature record cannot be due to its presence in the emissions record.

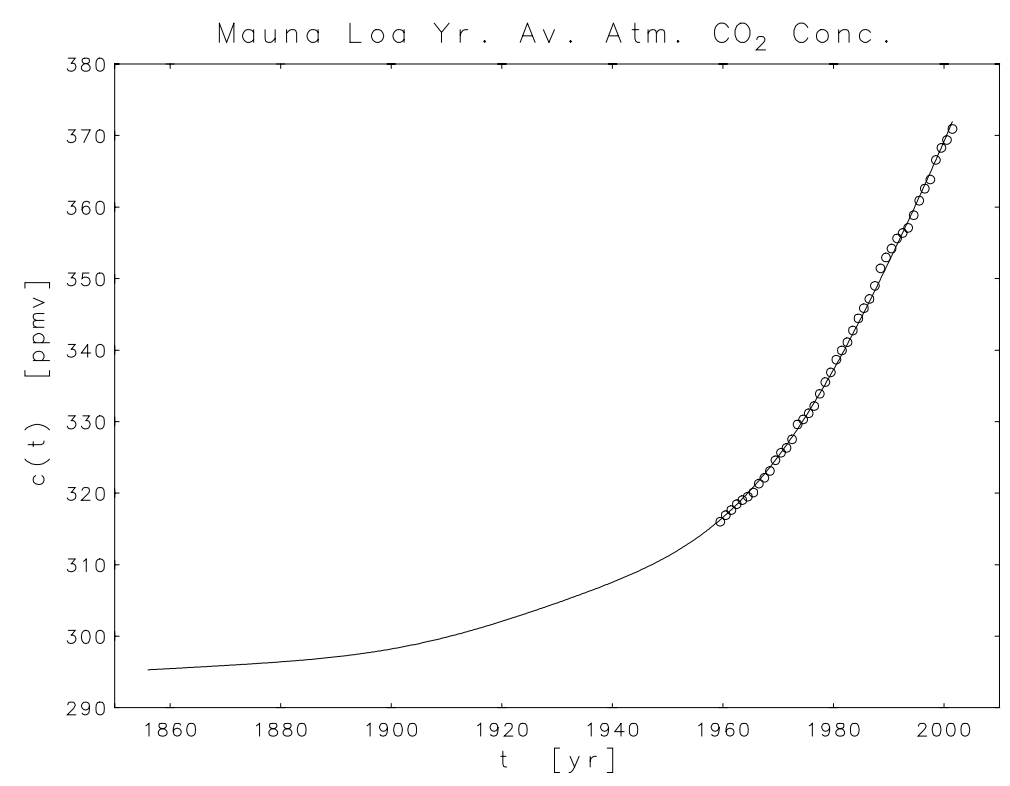

Fig. 3. The circles are the yearly average atmospheric $\mathrm{CO}_{2}$ concentrations in units of part per million by volume [ppmv] for the years 1959-2001. The curve was obtained by fitting (9) to the data and extending the fit back to 1856.0.

\section{Exercise 4: The Atmospheric Temperature Connection}

Conventional wisdom holds that the response of global temperatures to increasing atmospheric $\mathrm{CO}_{2}$ can only be estimated within the context of sophisticated climate models. But reconstructions [1,3] from Antarctican ice cores of atmospheric temperature changes and atmospheric $\mathrm{CO}_{2}$ concentrations for the past 160,000 years have shown that the two quantities have varied proportionately, and in lock step, through a temperature range of length $12.5^{\circ} \mathrm{C}$ and a concentration range of length $100 \mathrm{ppmv}$. This suggests a model of the form 
Table 2. Parameter estimates and coefficients of determination for three fits of (12) to the $\mathrm{CO}_{2}$ emissions, using temperature feedback models (6), (7), and (10).

\begin{tabular}{|c|rrr|}
\hline \hline Param. & Quad. Baseline(6) Expon. Baseline (7) $\mathrm{CO}_{2}$-driven Baseline (10) \\
\hline \hline$\hat{P}_{0}$ & $78.1 \pm 3.2$ & $93.5 \pm 3.7$ & $69.7 \pm 4.4$ \\
$\hat{\alpha}$ & $0.03862 \pm .00050$ & $0.03804 \pm .00051$ & $0.03758 \pm .00068$ \\
$\hat{\beta}$ & $1.481 \pm .053$ & $1.345 \pm .052$ & $0.994 \pm .053$ \\
\hline$R^{2}$ & 0.9963 & 0.9958 & 0.9930 \\
\hline \hline
\end{tabular}

$$
T\left(t-t_{0}\right)=T_{0}+C_{2} c\left(t-t_{0}\right)+A_{1} \sin \left[\frac{2 \pi}{64.9}\left(t-t_{0}-5.85\right)\right] .
$$

where $c\left(t-t_{0}\right)$ is the function plotted as a smooth curve in Fig. 3, and $T_{0}$, $C_{2}$, and $A_{1}$ are free parameters, with $T_{0}$ here being not $T\left(t_{0}\right)$ but rather the temperature anomaly for an atmosphere with no $\mathrm{CO}_{2}$. This model assumes that the 64.9 year cycle truly is a weather/climate effect that is superposed on the $\mathrm{CO}_{2}$-driven baseline. Fitting it to the temperature anomalies is another easy linear least squares problem, given the software written for the previous exercise. The parameter estimates are given in the last column of Table 1 and the fit is plotted as the coarsely dashed curve in Fig. 2.

\section{Exercises 5-7: The Gaian Feedback}

Since the 64.9 year temperature cycle is not caused by the $\mathrm{CO}_{2}$ emissions, the inverse cycle in the emissions must be caused by a negative, temperature dependent feedback. Rust and Kirk [13] modelled this feedback with the differential equation

$$
\frac{d P}{d t}=\left(\alpha-\beta \frac{d T}{d t}\right) P, \quad P\left(t_{0}\right)=P_{0},
$$

where $\alpha, \beta$, and $P_{0}$ were free parameters. With the shift of the zero point to $t_{0}$, the solution becomes

$$
P\left(t-t_{0}\right)=P_{0} \exp \left\{\alpha\left(t-t_{0}\right)-\beta\left[T\left(t-t_{0}\right)-T(0)\right]\right\} .
$$

Three exercises, which use the cylcle to confirm the feedback, are to fit this equation to the emissions data using the estimates from Table 1 in their corresponding models (6), (7), and (10) for $T\left(t-t_{0}\right)$. These are easy nonlinear least 


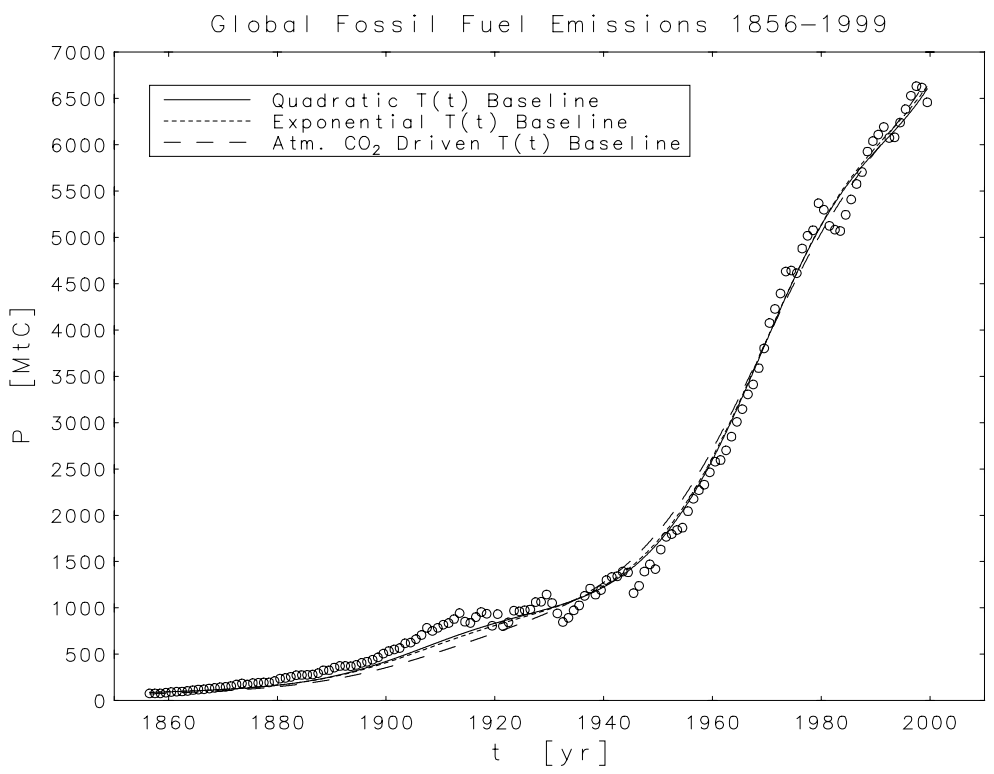

Fig. 4. Three temperature feedback model fits to the fossil fuel emissions record.

squares problems. The results are given in Table 2 and Fig. 4. None of the fits is quite as good as the solid curve in Fig. 11, which had $R^{2}=0.9973$, but all are good enough to lend strong support to the feedback hypothesis.

\section{Exercise 8: For Advanced Students}

With the fit of the $\mathrm{CO}_{2}$-driven version (10) of the model (12), in the last exercise above, the analysis has completed a full circle from the purely mathematical representation (21) for the emissions to the dynamical representation (11). The agreement between the two fits, i.e. between the solid curve in Fig. 1 and the coarsely dashed one in Fig. 4 is not perfect, but it is good enough to argue not only that the feedback hypothesis is correct, but also that the intermediate hypotheses (8) and (10) are least approximately correct. These results, taken together, suggest the following dynamical models for $P(t), c(t)$ and $T(t)$ :

$$
\begin{array}{lll}
\frac{d P}{d t}=\alpha P-\beta\left\{\eta P+A \cos \left[\frac{2 \pi}{\tau}(t+\phi)\right]\right\} P, & P\left(t_{0}\right)=P_{0}, \\
\frac{d c}{d t}=\gamma P & , \quad c\left(t_{0}\right)=c_{0}, \\
\frac{d T}{d t}=\eta P+A \cos \left[\frac{2 \pi}{\tau}(t+\phi)\right] & T\left(t_{0}\right)=T_{0},
\end{array}
$$

with unknown parameters $\alpha, \beta, \gamma, \eta, A, \tau, \phi, P_{0}, c_{0}$, and $T_{0}$. The exercise, left to the student, is to fit these three equations simultaneously to the three data sets. 


\section{References}

1. Barnola, J. M., Raynaud, D., Korotkevich, Y. S., Lorius, C.: Vostok ice core provides 160,000-year record of atmospheric $\mathrm{CO}_{2}$. Nature, 329 (1987) 408-414

2. Jones, P. D., Moberg, A.: Hemispheric and large-scale surface air temperature variations: An extensive revision and an update to 2001. Jour. Climate, 16 (2003) 206-223

3. Jouzel, J., Lorius, C., Petit, J. R., Genthon, C., Barkov, N. I., Kotlyakov, V. M., Petrov, V. M.: Vostok ice core: a continuous isotope temperature record over the last climatic cycle (160,000 years). Nature, 329 (1987) 403-408

4. Keeling, C. D., Whorf, T. P.: Atmospheric carbon dioxide record from Mauna Loa. in Online Trends: A Compendium of Data on Global Change, http://cdiac.ornl.gov/trends/co2/contents.htm, Carbon Dioxide Information Analysis Center, Oak Ridge National Laboratory, Oak Ridge, TN (2003)

5. Lovelock, J. E., Margulis, L.: Atmospheric homeostasis by and for the biosphere: the gaia hypothesis. Tellus, 26 (1974) 2-10

6. Marland, G., Boden, T. A., Andres, R. J.: Global, Regional, and National $\mathrm{CO}_{2}$ Emissions. in Trends: A Compendium of Data on Global Change, Carbon Dioxide Information Analysis Center, Oak Ridge National Laboratory, Oak Ridge, TN (2000)

7. Rust, B. W.: Fitting nature's basic functions Part I: polynomials and linear least squares. Computing in Science \& Engineering, 3 nr 5 (2001) 84-89

8. Rust, B. W.: Fitting nature's basic functions Part II: estimating uncertainties and testing hypotheses. Computing in Science \& Engineering, 3 nr 6 (2001) 60-64

9. Rust, B. W.: Fitting nature's basic functions Part III: exponentials, sinusoids, and nonlinear least squares. Computing in Science \& Engineering, 4 nr 4 (2002) 72-77

10. Rust, B. W.: Fitting nature's basic functions Part IV:the variable projection algorithm. Computing in Science \& Engineering, 5 nr 2 (2003) 74-79

11. Rust, B. W.: Separating signal from noise in global warming. Computing Science and Statistics, 35 (2003) in press

12. Rust B. W., Crosby, F. J.: Further studies on the modulation of fossil fuel production by global temperature variations. Environment International, 20 nr 4 (1994) 429-456

13. Rust B. W., Kirk, B. L.: Modulation of fossil fuel production by global temperature variations. Environment International, 7 (1982) 419-422

14. Schlesinger, M. E., Ramankutty, N.: An oscillation in the global climate system of period 65-70 years. Nature, 367 (1994) 723-726

15. Watts, J. A.: The Carbon Dioxide Question: Data Sampler. in Clark, W. C., editor, Carbon Dioxide Review: 1982, Oxford University Press, New York (1982) 431-469 